\title{
A High-quality Genome Assembly and Annotation of Quercus Acutissima Carruth
}

\section{Dan Liu}

Beijing Forestry University

\section{Chengcheng Zhou}

Beijing Forestry University

\section{Boqiang Tong}

Shandong Province Center of Forest and Germplasm Resources

Xiaoman Xie

Shandong Province Center of Forest and Germplasm Resources

\section{Haiping Yang}

Shandong Province Center of Forest and Germplasm Resources

\section{Biao Han}

Shandong Province Center of Forest and Germplasm Resources

\section{Yizeng Lu}

Shandong Province Center of Forest and Germplasm Resources

Wei Li ( $\sim$ bjfuliwei@bjfu.edu.cn )

Beijing Forestry University

\section{Wenqing Li}

Shandong Province Center of Forest and Germplasm Resources

\section{Research Article}

Keywords: Quercus acutissima Carruth, Oaks, Genome assembly and annotation

Posted Date: February 14th, 2022

DOI: https://doi.org/10.21203/rs.3.rs-1290824/v1

License: (c) (1) This work is licensed under a Creative Commons Attribution 4.0 International License. Read Full License 


\section{Abstract}

Background: Quercus acutissima is an economic and ecological tree species often used for afforestation of arid and semi-arid lands and is considered an excellent tree species for soil and water conservation. However, the $Q$. acutissima genome sequencing and assembly have not been reported, which limiting molecular biology studies and breeding progress in Q. acutissima.

Results: We generated a $956.9 \mathrm{Mb}$ genome with a contig N50 of 1,203,427 bp and 53,304 annotated genes. Meanwhile, the assembled contigs $(1,507)$ assigned to a total of 12 chromosomes. The BUSCO database evaluation accounted for $98.0 \%$ of the genome size and the genome heterozygous rate was $0.25 \%$. Repetitive sequences constituted $57.13 \%$ of the assembled genome, the most abundant one was long terminal repeats ( $7.18 \%$ of the genome). Phylogenetic analysis showed that Q. acutissima and Fagus sylvatica were differentiated around 54.2 million years ago (Mya), and Q. sober was differentiated around 4.4 Mya.

Conclusion: We sequenced, assembled and annotated the first high-quality genome of $Q$. acutissima. This work not only promises to accelerate molecular biology studies and breeding progress in $Q$. acutissima, Q. acutissima is one of the important species in Quercus, but also promote evolutionary studies at the genome level in the genus Quercus due to its importance as one of the important species in Quercus.

\section{Background}

The genus Quercus (oaks), as one of the largest genus in Fagaceae, contains more than 400 species widely distributed in Asia, Europe, Africa and North America [1]. The uses of oaks are various, including timber, bioenergy, and dyes production [2-4]. After the progressive development from plastid DNA and nuclear DNA sequence to genomics datasets [5-7], two subgenus, Quercus and Cerris, have been divided from the Quercus according to the molecular classification method [8]. The Quercus includes five groups (sections): Ponticae, Virentes, Protobalanus (intermediate Oaks), Quercus (white oaks), and Lobatae (red oaks). The Cerris includes three groups (sections): Ilex, Cerris and Cyclobalanopsis. Within the Quercus genus, evolutionary profiles of plastid genomes in some species have been elucidated, including $Q$. acutissima, Q. aliena, Q. aquifolioides, Q. baronii, Q. dolicholepis, Q. edithiae, Q. fabri, Q. glauca and 10 other Quercus plastomes [9]. However, there are still relatively few species with complete whole gene sequencing, including $Q$. lobata [10], Q. suber [11], and Q. robur [12], which obstacles the research process of whole-genome systematic evolution and gene function analysis of the Quercus.

As an ecological and economic tree species, Quercus acutissima widely distributed in East Asia, especially in southeast China (latitude from $18^{\circ}$ to $41^{\circ} \mathrm{N}$ and longitude from $91^{\circ}$ to $123^{\circ} \mathrm{E}$ ) [3,13]. The silviculture of $Q$. acutissima is usually mixed and secondary monocultures, which are also distributed in a scattered manner in harsh environments, and its hardwoods provide excellent building material $[14,15]$. The timber of $Q$. acutissima is also used for charcoal production, which is a necessary fuel for daily life in 
many Asian countries, including China, Japan, Korea14. In northern China, Q. acutissima forest ecosystems have been destroyed due to human disturbance. $Q$. acutissima genetic resources are at risk $[14,15]$. Thus, planning breeding and conservation program for $Q$. acutissima native populations is crucial, and the genome-wide evolution, gene function and molecular breeding are important approaches to support this goal [16]. At present, related research on $Q$. acutissima mainly focus on rapid propagation technology, seedling growth characteristics, genetic selection of excellent families and studies related to population genetic diversity. To date, the $Q$. acutissima genome sequencing and assembly have not been reported.

In our study, Q. acutissima genome was sequenced and assembled using Illumina, PacBio, Hi-C sequencing and de novo assembly. We expect that this study will facilitate whole-genome evolutionary and comparative and functional genomics of $Q$. acutissima studies. Our results improved our understanding of the evolution and diversification of duplicated genes in $Q$. acutissima, laying a vital foundation for the discovery and research of novel genes contributing to excellent properties in breeding programs.

\section{Results}

\section{Genome sequencing and assembly}

In Q. acutissima genome sequencing, the sequencing depth was 99x and a total of $154.41 \mathrm{~Gb}$ reads were obtained using PacBio sequencing. The subread N50 was 24,256 bp (Table 1). The k-mer value was 21 in genome size estimation and the frequency curve displayed a Poisson distribution (Figure S1). Genomic heterozygosity and duplication resulted in the highest curve peak was at a depth of 60 (depth > 30). The genome size and heterozygosity of Q. acutissima were estimated to be $1.38 \mathrm{~Gb}$ and $0.25 \%$, respectively. These results indicated that Q. acutissima genome was large with low heterozygous.

Table 1. Statistics of PacBio Sequel sequencing data.

\begin{tabular}{|ll|}
\hline Index & PacBio \\
\hline Subreads number & $8,972,662$ \\
\hline Subreads Average Mean Length(bp) & $17,209.06$ \\
\hline Subreads N50(bp) & 24,256 \\
\hline Subreads Read Base(bp) & $154,411,063,662$ \\
\hline GC mean & 0.38 \\
\hline
\end{tabular}

We used a series of analytical methods and software for the species genome assembly, and an estimated genome size was $957,596,940$ bp in first assembly, with a contig N50 of 1,204,852 bp. After the third- 
generation subreads assembly and the genome map correction, the corrected genome size was $956,937,475 \mathrm{bp}$, with a contig N50 of 1,203,427 bp (Table 2). Among the obtained sequences, $98.00 \%$ were anchored to 12 chromosomes (Figure 1).

Table 2. Summary of Quercus acutissima genome assembly.

\begin{tabular}{|lll|}
\hline Items & Contigs length(bp) & Contigs number \\
\hline Total & $956,937,475$ & 1,507 \\
\hline Max & $8,261,252$ & - \\
\hline Number>=2000bp & - & 1,506 \\
\hline N50 & $1,203,427$ & 223 \\
\hline N60 & 984,567 & 310 \\
\hline N70 & 766,315 & 421 \\
\hline N80 & 517,641 & 574 \\
\hline N90 & 282,007 & 809 \\
\hline
\end{tabular}

\section{Assessment of genomic integrity}

The completeness and accuracy of the genome assembly and gene data sets were evaluated using BUSCO software. Comparison analysis between gene data sets and genome showed that the genome assembly completeness was as expected ( $98 \%$ of complete BUSCOs; Table S1). High-quality genomes do not show significant dispersion in their GC distribution and sequencing depth distribution. Therefore, GCdepth analysis showed that there was no obvious left-right chunking in the GC-depth plot (Figure S2) and the average GC content was $35.18 \%$ (see Table S2, for other base pairs contents), indicating no contamination and high assembly quality of assembled genome. Additionally, we found that the genomic reads and coverage were deep and complete by comparing the sequencing reads to the assembled config sequences (Figure S3). The comparison analysis of the next-generation data showed $99.84 \%$ of comparison proportion identified the genome assembly high quality (Table S3).

\section{Genome annotation}

We used RepeatModeler software to establish a de novo repeat sequence library, and then used RepeatMasker and RepeatproteinMask software to predict the genome sequence. The two software programs revealed a total length of $95,429,294 \mathrm{bp}$ and $146,533,493 \mathrm{bp}$, representing $9.97 \%$ and $15.31 \%$ of the genome, respectively. In addition, The total length of tandem repeats obtained by the de novo prediction method was $47,779,176 \mathrm{bp}$, representing $4.99 \%$ of the genome. When the overlapping portions of the non-redundant repeats were deleted, a total of $546,670,373 \mathrm{bp}$ of non-redundant repeats were identified, which accounting for $57.13 \%$ of the assembled genome (Table S4). In the repeat sequences 
analysis, LTRs accounted for $7.18 \%$ of total repeat sequences (Supplementary Table S5). Additionally, we identified miRNA, snRNA and rRNA genes and predicted tRNAs and rRNAs, resulting in a $Q$. acutissima genome with 529 miRNAs, 948 tRNAs, 1,061 rRNAs and 279 snRNAs (Table S6).

Additionally, a total of 55,149 predicted genes were generated by analyzing the potential of the predicted genes. Their average lengths were $3,828.07 \mathrm{bp}$, and the average coding sequences was $1130.91 \mathrm{bp}$. Based on the comparison between predicted gene sets with the annotation databases, $96.65 \%$ of the genome genes were annotated (Table S7, Fig. 2). These annotated statistics were statistically showed in the Figure 2.

\section{Gene family and phylogenetic relationships}

Gene families analysis by OrthoMCL software identified 337 specific genes of $Q$. acutissima (Table S8). The genes of $Q$. acutissima produced more numbers in common with $Q$. suber and $Q$. lobata than with the other species (Figure S4). According to 4DTv sites value of orthologous family genes, the species phylogenetic tree (ML-Tree) was constructed using PhyML software after the integrated supergene sequence was constructed. As shown in Figure 3, Q. robur and Q. lobata in Fagaceae diverged from one another earlier than $Q$. acutissima, and $Q$. acutissima is most closely related to $Q$. suber. According to above results, we estimated divergence time by using PAML's MCMCTREE software. The corrected time directly obtained from TimeTree website (http://timetree.org/). The result showed that the divergence time between Casuarinaceae and Fagaceae was 90 Ma.

\section{Wholegenome duplication and gene families expansion/contraction analysis}

In the gene families expansion and contraction analysis (Figure 4a), we found 11709 gene families in the MCRA (most recent common ancestor). In comparison to Q. lobata, Q. suber, Q. robur, C. equisetifolia and F. sylvatica, there were 428 and 176 gene families showing expansion and contraction in Q. acutissima, respectively (Table S9). Additionally, GO enrichment analysis revealed similar trends in the distribution of expansion and contraction in gene families across all GO terms (Figure 4b), suggesting that dynamic changes in gene function are due to a combination of gene family expansion and contraction.

In plants, WGD events are widespread and play a vital role for plant genome evolution [17]. After multiple sequence alignment of sequences in the synteny blocks within $Q$. acutissima and other species, 4DTv site values were calculated. The relative timing of WGD was estimated based on these values. In general, two significant peaks were seen in the $Q$. acutissima genome with $Q$. suber and $F$. sy/vatica, respectively (Figure 5a), suggesting that $Q$. acutissima has experienced two WGD events. Additionally, we identified one ancient WGD events in Q. suberwith one peak at $\sim 0.01$ and 1.18.

To explore the evolutionary source of chromosomes among $Q$. acutissima, $Q$. robur, and $Q$. lobata, the aligned protein sequences among three species ( $Q$. acutissima, Q. robur, and Q. lobata) were obtained by BLASTP. Based on these aligned results, we used MCScan to evaluate genome synteny blocks, suggesting that $Q$. acutissima and $Q$. robur displayed a closely genomic synteny relationship (Figure $5 b$ ). 


\section{Discussion}

Q. acutissima, Fagaceae, is an economically and ecologically important tree species with wide distribution in China $[18,19]$. Here, we generated the first $Q$. acutissima draft genome at the chromosomelevel. The assembled genome size is roughly $956.9 \mathrm{Mb}$, with contig N50 sizes of 1,203,427 bp. The development of PacBio sequencing has resulted in a considerable increase in contig N50 sizes compared to previous sequencing technologies [20]. The contig N50 sizes of Q. lobata was 18,900 bp just using Illumina sequencing [10], which is much shorter than contig N50 length of our genome. Thus, the assemble length of contig N50 sizes can represent the genome assembling quality [21], consequently, our genome has high assembly contiguity. High heterozygosity and repetition rates are responsible for the inability to assemble high-quality genomes $[22,23]$. The heterozygous rate of $Q$. acutissima was $0.25 \%$, which is lower than that of Q. lobata (1.25\%)10 and Q. suber (1.62\%)11. It is worth noting that $98 \%$ of complete BUSCO core genes were detected in the assembly genome, which is higher than that of Q. lobata (94\%)10 and comparable to Q. suber genome (97\%)11 that were built with sequences from four different platforms. In brief, the assembly of $Q$. acutissima is relatively accurate and complete, which will provide a valuable genome resource for understanding the evolution, active ingredient biosynthesis, and genetic improvement of this species.

The genus Quercus (Fagaceae), which includes nearly 300 species, is distributed in Asia, Africa, Europe and North America [24]. But there are still relatively few reports on genome research in this genus. In our study, a total of 53,304 genes were annotated by different databases, accounting for $96.65 \%$ of total genes (Table S7), indicating that there are a lot of genes in Quercus worthy of further research and identification. As a member in this genus, $Q$. acutissima genome information can fill genome research gap and promote the evolutionary biology research in Quercus. Following the statistical analysis of repeat sequences in the genome, we found that the repeat regions accounted for $57.13 \%$ of the genome, the numbers of repetitive sequences and ncRNA sequences were relatively high in $Q$. acutissima compared with Quercus other species. To understand the evolutionary status of $Q$. acutissima, we analyzed its evolution and divergence times. The 4DTv analysis found that $Q$. acutissima shared two ancient WGD event with $Q$. suber and F. sylvatica (Fig. 5a). WGD events can lead to genome size variation, gene families expansion and contraction, chromosomal rearrangement, and species evolution in plant $[22,25]$. We found a high collinearity relationship between $Q$. acutissima and $Q$. robur chromosomes, which may be resulting from the two species have not experienced recent WGD events after species divergence.

To sum up, we obtained high-quality genome sequences of $Q$. acutissima using Pacbio sequencing, Illumina sequencing, $\mathrm{Hi}-\mathrm{C}$ technology and de novo assembly. The development of sequencing technologies, analytical methods, and statistical algorithms continue to promote the efficiency and accuracy of genome sequencing and assembly $[17,20,26]$. The $Q$. acutissima genome includes a high quality chromosomal-level assembly and many important genes, offering novel insights into genome evolution, functional innovation and key regulatory pathways in wood formation and production of high- 
value metabolites, and providing excellent genetic resources for comparative genome studies among Quercus species.

\section{Materials And Methods}

\section{Plant materials, DNA extraction and genome sequencing}

Fresh leaves of $Q$. acutissima tree growing in the Shandong Forestry and Grass Germplasm Resources Center were immediately frozen in liquid nitrogen and stored at $-80^{\circ} \mathrm{C}$ until DNA was extracted, and all plant materials were preserved at Shandong Forestry and Grass Germplasm Resources Center. Total genomic DNA was extracted and purified from leaf tissue according to phenol chloroform isoamyl alcohol method. After obtaining high-quality purified genomic DNA samples, PCR free SMRT bell library was constructed and sequenced by PacBio sequel/sequel II sequencing platform. Finally, we obtained $154.41 \mathrm{~Gb}$ of subreads with $99 \times$ coverage of the $Q$. acutissima genome. We constructed a paired-end library with an insert size of $350 \mathrm{bp}$ and sequenced using the Illumina HiSeq X Ten platform.

\section{Genome assembly, quality evaluation and chromosome anchoring}

Before $Q$. acutissima genome de novo assembly, the genome size was estimated using the k-mer distribution assessment $(\mathrm{k}=21)$ using $1.38 \mathrm{~Gb}$ of high-quality paired-end reads from Illumina short reads. Sequences assembly is to use analysis software, including Canu [27], FALCON [28], and SmartDenovo [29], to take the long sequence Reads generated after error correction, construct and filter reliable overlap relations by comparing them, and finally construct reliable overlap group (contigs) sequences based on the overlap relation map, which produced assembly agglomerations containing 1,507 contigs for the preliminary assembly. Owing to the high heterozygosity of the $Q$. acutissima genome, the clean PacBio subreads were aligned back to the assembled contigs using BLASR (v5.1) [30] and Arrow software (v2.2.1, https://github.com/PacificBiosciences/Genomic-Consensus) with default parameters. After the assembly of the third generation subreads, there will be a certain amount of error information such as short Indel and SNP due to the presence of sequencing errors. Based on the second-generation data, the second-generation Clean Reads will be compared to the third-generation corrected sequences using BWA (v0.7.9a, parameter, $-\mathrm{k}$ 30) [31] and Pilon software (v1.22, default parameters) [32]. In addition, based on the OrthoDB [33] database, we performed a BUSCO (version 3.0.1) [34] assessment using single-copy orthologous genes to confirm the quality of the genome assembly.

Fresh leaves of $Q$. acutissima were used to construct a Hi-C sequencing library. The library was then sequenced using the Illumina platform, and the clean sequences were mapped to the draft genome using BWA, with valid $\mathrm{Hi}-\mathrm{C}$ reads employed to correct the draft assembly. Finally, the draft genome of $Q$. acutissima was assembled into chromosomes.

Genome annotation 
Repeated sequences are an important part of the genome and consist of two main categories: Tandem Repeat and Interspersed Repeat, respectively. We used RepeatModeler software [35] to build the De novo repeat sequence library. Subsequently, we aligned the genome sequences to Repbase [36] by using RepeatMasker [37] for the searching of homologous repeat sequences. Homology prediction were conducted by comparing homologous proteins from plant genomes, including Quercus lobata, Quercus suber, Quercus robur, Fagus sy/vatica and Casuarina equisetifolia, with Q. acutissima genomic sequences using Blast (E-value cutoff : 1e-5; http://blast.ncbi.nlm.nih.gov/Blast.cgi) and the GeneWise web resource (version 2.2.0) [38]. Gene structure models were predicted by comparing EST/cDNA sequences with genome using PASA software (version 2.3.3) [39]. Combining the above forecast results, using the evincemodeler (EVM) [40] integrates the gene set predicted by various strategies into a nonredundant and more complete gene set (Supplementary Table S10).

We used the NCBI protein database, GO [41], KEGG [42], NR

(ftp://ftp.ncbi.nlm.nih.gov/blast/db/FASTA/nr.gz), PFAM [43], eggNOG-mapper [44] and others annotated the gene function.

\section{Expansion/contraction analysis of gene families}

The genomes of $Q$. acutissima and five other plants, including Q. lobata, Q. suber, Q. robur, Casuarina equisetifolia and Fagus sy/vatica, were collected for evolutionary analysis. The software OrthoMCL [45] was used to construct a phylogenetic tree for all protein sequences. Multiple sequence alignment among the synteny blocks sequences and the 4DTv value calculation were conducted using MUSCLE [46]. Divergence times were estimated based on the supergene sequences integrated in the phylogenetic analysis using the mcmctree software [47] in the PAML [48] package with the BRMC method [49,50], and the correction times were taken from the TimeTree [51].

\section{Gene families and phylogenetic analysis}

Based on the clustering analysis of gene families, gene family expansion and contraction analysis were performed using CAFÉ [52], using PGM (probabilistic graphical models) models for phylogenetic trees, simulating gene acquisition and loss under specified evolutionary trees, and by hypothesis testing.

\section{Synteny and WGD analysis}

To find the synteny blocks between $Q$. lobata, $Q$. robur and $Q$. acutissima, the python version of MCScan (version 0.8 ) $[53,54]$ was applied to compare proteins to proteins. The circular plot of the $Q$. acutissima genome was produced using Circos [55]. The period when whole genome duplication occurred in the species and the period of divergence between the target and comparison species were estimated based on the abundance of $4 \mathrm{dTV}$ values.

\section{Declarations}

\section{Declaration statements}


We confirm that all methods in our research were performed in accordance with the relevant guidelines and regulations, and that the collection and preservation of all plant materials with permission from Shandong Forestry and Grass Germplasm Resources Center, and that the authors comply with the IUCN Policy Statement on Research Involving Species at Risk of Extinction and the Convention on the Trade in Endangered Species of Wild Fauna and Flora.

All plant materials collected by the first author Dan Liu on March 22, 2020 at Shandong Forest and Germplasm Resources Center. The first author Dan Liu undertook the formal identification of the plant material used in our study and all plant materials have been deposited in Shandong Forest and Germplasm Resources Center in China.

\section{Acknowledgments}

We are grateful for the generous grant from the National Engineering Laboratory of Tree breeding of Beijing Forestry University and Shandong Province Center of Forest and Germplasm Resources that made this work possible.

\section{Authors' Contributions}

WL and WQL designed and supervised the study. DL, CCZ, BQT, XMX, and HPY collected the samples and extracted the genomic DNA and RNA. DL, CCZ, B.H, and HPY performed genome assembly and bioinformatics analysis. DL and CCZ wrote the original manuscript. WL and WQL reviewed and edited this manuscript. All authors read and approved the final manuscript.

\section{Funding}

This research was funded by the 'Collection, Conservation, and Accurate Identification of Forest Tree Germplasm Resources' of Shandong Provincial Agricultural Elite Varieties Project, grant number 2019LZGC018; Biosafety and Genetic Resources Management Project of State Forestry and Grassland Administration, grant number KJZXSA202111; Project of National Forest Germplasm Resources Sharing Service Platform Construction and Operation, grant number 2005-DKA21003.

\section{Availability of data and materials}

The data that support the findings of this study have been deposited into CNGB Sequence Archive (CNSA) [56] of China National GeneBank DataBase (CNGBdb) [57] with accession number CNP0002369, other data referred to are included in the manuscript or supplementary materials.

\section{Ethics approval and consent to participate}

Not applicable.

\section{Consent for publication}


Not applicable.

\section{Competing interests}

The authors declare that they have no competing interests.

\section{References}

1. Li M, Guo W, Du N, et al. Nitrogen deposition does not affect the impact of shade on Quercus acutissima seedlings. PLoS ONE. 2018;13:e194261.

2. Sasaki C, Kushiki Y, Asada C, et al. Acetone-butanol-ethanol production by separate hydrolysis and fermentation (SHF) and simultaneous saccharification and fermentation (SSF) methods using acorns and wood chips of Quercus acutissima as a carbon source. Ind Crop Prod. 2014;62:286-292.

3. Li X, Li Y, Zang M, et al. Complete chloroplast genome sequence and phylogenetic analysis of Quercus acutissima. Int J Mol Sci. 2018;19:2443.

4. Wu T, Wang GG, Wu Q, et al. Patterns of leaf nitrogen and phosphorus stoichiometry among Quercus acutissima provenances across China. Ecol Complex. 2014;17:32-39.

5. Deng M, Jiang X, Hipp AL, et al. Phylogeny and biogeography of East Asian evergreen oaks (Quercus section Cyclobalanopsis Fagaceae): Insights into the Cenozoic history of evergreen broad-leaved forests in subtropical Asia. Mol Phylogenet Evol. 2018;119:170-181.

6. Hipp AL, Manos PS, González RA, et al. Sympatric parallel diversification of major oak clades in the Americas and the origins of Mexican species diversity. New phytol. 2018;217:439-452.

7. Denk T, Grimm GW. The oaks of western Eurasia: traditional classifications and evidence from two nuclear markers. Taxon 2010;59:351-366.

8. Denk T, Grimm GW, Manos PS, et al. An updated infrageneric classification of the oaks: review of previous taxonomic schemes and synthesis of evolutionary patterns. M//Cham: Springer International Publishing 2017 13-38.

9. Li X, Li Y, Sylvester SP, et al. Evolutionary patterns of nucleotide substitution rates in plastid genomes of Quercus. Ecol Evol. 2021;11:13401-13414.

10. Sork VL, Fitz-Gibbon ST, Puiu D, et al. First draft assembly and annotation of the genome of a california endemic oak Quercus lobata Née (Fagaceae). G3 (Bethesda Md.) 2016;6:3485-3495.

11. Ramos AM, Usié A, Barbosa P, et al. The draft genome sequence of cork oak. Sci Data. 2018;5:180069.

12. Plomion C, Aury J, Amselem J, et al. Decoding the oak genome: public release of sequence data assembly annotation and publication strategies. Mol Ecol Resour. 2016;16:254-265.

13. Yang $B, H e F$, Zhao X, et al. Composition and function of soil fungal community during the establishment of Quercus acutissima (Carruth.) seedlings in a Cd-contaminated soil. Environ Manage. 2019;246:150-156. 
14. Zhang YY, Fang YM, Yu MK, et al. Molecular characterization and genetic structure of Quercus acutissima germplasm in China using microsatellites. Mol. Biol. Rep. 2013;40:4083-4090.

15. Aldrich PR, Parker GR, Ward JS, et al. Spatial dispersion of trees in an old-growth temperate hardwood forest over 60 years of succession. Forest Ecol. Manage. 2003;180:475-491.

16. Greene SL, Morris JB. The case for multiple-use plant germplasm collections and a strategy for implementation. Crop Sci. 2001;41:886-892.

17. Xue $T$, Zheng $X$, Chen $D$, et al. A high-quality genome provides insights into the new taxonomic status and genomic characteristics of Cladopus chinensis (Podostemaceae). Hortic. Res. 2020;7:46.

18. Zhang RS, Yang J, Hu HL, et al. A high level of chloroplast genome sequence variability in the Sawtooth Oak Quercus acutissima. Int. J. Biol. Macromol. 2020;152:340-348.

19. Li X, Li Y, Zang M, et al. Complete chloroplast genome sequence and phylogenetic analysis of Quercus acutissima. Int. J. Mol. Sci. 2018;19:2443.

20. Wei S, Yang Y, Yin T. The chromosome-scale assembly of the willow genome provides insight into Salicaceae genome evolution. Hortic. Res. 2020;7:45.

21. Yang C, Ma L, Xiao D, et al. Chromosome-scale assembly of the Sparassis latifolia genome obtained using long-read and Hi-C sequencing. G3 (Bethesda Md.). 2021;11:jkab173.

22. Wang J, Xu S, Mei Y, et al. A high-quality genome assembly of Morinda officinalis a famous native southern herb in the Lingnan region of southern China. Hortic. Res. 2021;8:135.

23. Gao S, Wang B, Xie S, et al. A high-quality reference genome of wild Cannabis sativa. Hortic. Res. 2020;7:73.

24. Bent JS. Quercus: Classification Ecology and Uses. Nova Science Publishers Inc. 2020.

25. El BM, Panaud O. Comparative genomic paleontology across plant kingdom reveals the dynamics of TE-driven genome evolution. Genome Biol. Evol. 2013;5:954-965.

26. Wu S, Sun W, Xu Z, et al. The genome sequence of star fruit (Averrhoa carambola). Hortic Res. 2020;7:95.

27. Koren S, Walenz BP, Berlin K, et al. Canu: scalable and accurate long-read assembly via adaptive kmer weighting and repeat separation. Genome Res. 2017;27:722-736.

28. Chin CS, Peluso P, Sedlazeck FJ, et al. Phased diploid genome assembly with single-molecule realtime sequencing. Nat. methods. 2016;13:1050.

29. Istace $B$, Friedrich $A$, Agata $L$, et al. de novo assembly and population genomic survey of natural yeast isolates with the Oxford Nanopore MinION sequencer. Gigascience. 2017;6:1-13.

30. Chaisson MJ, Tesler G. Mapping single molecule sequencing reads using basic local alignment with successive refinement (BLASR): application and theory. BMC Bioinformatics. 2012;13:238.

31. Li H, Durbin R. Fast and accurate short read alignment with Burrows-Wheeler transform. Bioinformatics. 2009;25:1754-1760.

32. Walker BJ, Abeel T, Shea T, et al. Pilon: an integrated tool for comprehensive microbial variant detection and genome assembly improvement. PLoS ONE. 2014;9:e112963. 
33. Kriventseva EV, Kuznetsov D, Tegenfeldt F, et al. OrthoDB v10: sampling the diversity of animal plant fungal protist bacterial and viral genomes for evolutionary and functional annotations of orthologs. Nucleic Acids Res. 2019;47:D807-D811.

34. Simão FA, Waterhouse RM, loannidis P, et al. BUSCO: assessing genome assembly and annotation completeness with single-copy orthologs. Bioinformatics. 2015;31:3210-3212.

35. Flynn JM, Hubley R, Goubert C, et al. RepeatModeler2 for automated genomic discovery of transposable element families. Proc. Natl. Acad. Sci. 2020;117:9451-9457.

36. Jurka J, Kapitonov VV, Pavlicek A, et al. Repbase Update a database of eukaryotic repetitive elements. Cytogenet. Genome Res. 2005;110:462-467.

37. Tarailo-Graovac M, Chen N. Using RepeatMasker to identify repetitive elements in genomic sequences. Current Protocols in Bioinformatics. 2009;4:4-10.

38. Birney E, Clamp M, Durbin R. GeneWise and Genomewise. Genome Res. 2004;14:988-995.

39. Roberts A, Pimentel $H$, Trapnell $C$, et al. Identification of novel transcripts in annotated genomes using RNA-Seq. Bioinformatics. 2011;27:2325-2329.

40. Haas BJ. Salzberg SL, Zhu W, et al. Automated eukaryotic gene structure annotation using EVidenceModeler and the Program to Assemble Spliced Alignments. Genome Biol. 2008;9:R7.

41. Mi H, Huang X, Muruganujan A, et al. PANTHER version 14: more genomes a new PANTHER GO-slim and improvements in enrichment analysis tools. Nucleic Acids Res. 2019;47:D419-D426.

42. Kanehisa $M$, Sato $Y$, Kawashima M, et al. KEGG as a reference resource for gene and protein annotation. Nucleic Acids Res. 2016;44:D457-D462.

43. Finn RD, Bateman A, Clements J, et al. Pfam: the protein families database. Nucleic Acids Res. 2014;42:D222-D230.

44. Cantalapiedra CP, Hernández-Plaza A, Letunic I, et al. eggNOG-mapper v2: functional annotation orthology Assignments and domain prediction at the metagenomic scale. Mol. Biol. Evol. 2021:msab293.

45. Li L, Stoeckert JCJ, Roos DS. OrthoMCL: identification of ortholog groups for eukaryotic genomes. Genome Res. 2003;13:2178-2189.

46. Edgar RC. MUSCLE: multiple sequence alignment with high accuracy and high throughput. Nucleic Acids Res. 2004;32:1792-1797.

47. Yang Z. PAML 4: Phylogenetic analysis by maximum likelihood. Mol. Biol. Evol. 2007;24:1586-1591.

48. Guindon SDJLV. New algorithms and methods to estimate maximum-likelihood phylogenies: assessing the performance of PhyML 3.0. Syst. Biol. 2010;3:307-321.

49. Blanc G, Wolfe KH. Widespread paleopolyploidy in model plant species inferred from age distributions of duplicate genes. The Plant Cell 2004;16:1667-1678.

50. Sanderson MJ. r8s: inferring absolute rates of molecular evolution and divergence times in the absence of a molecular clock. Bioinformatics. 2003;19:301-302. 
51. Kumar S, Stecher G, Suleski M, et al. TimeTree: a resource for timelines timetrees and divergence times. Mol. Biol. Evol. 2017;34:1812-1819.

52. De Bie T, Cristianini N, Demuth JP, et al. CAFE: a computational tool for the study of gene family evolution. Bioinformatics. 2006;22:1269-1271.

53. Huang S, Li R, Zhang Z, et al. The genome of the cucumber Cucumis sativus L. Nat. Gentic. 2009;41:1275.

54. Schmutz J, Cannon SB, Schlueter J, et al. Genome sequence of the palaeopolyploid soybean. Nature. 2010;463:178.

55. Krzywinski M, Schein J, Birol I, et al. Circos: an information aesthetic for comparative genomics. Genome Res. 2009;19:1639-1645.

56. Guo XQ, Chen FZ, Gao F, Li L, Liu K, You LJ, et al. CNSA: a data repository for archiving omics data. Database (Oxford). 2020:baaa055.

57. Chen FZ, You LJ, Yang F, Wang LN. CNGBdb: China National GeneBank DataBase. Hereditas. 2020;42:799-809.

\section{Figures}




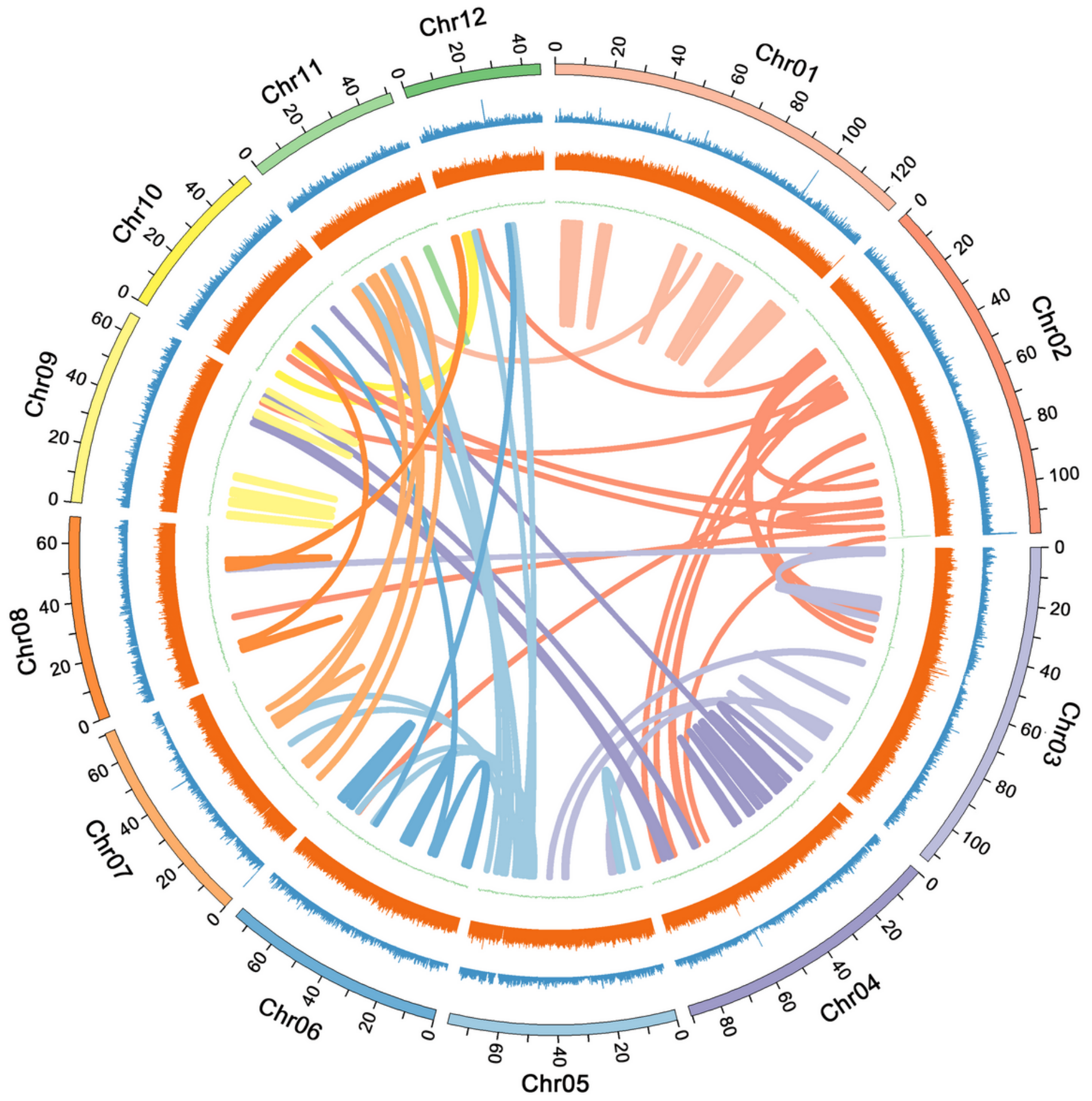

Figure 1

The topography and synteny of $Q$. acutissima genome. 


\section{NR}

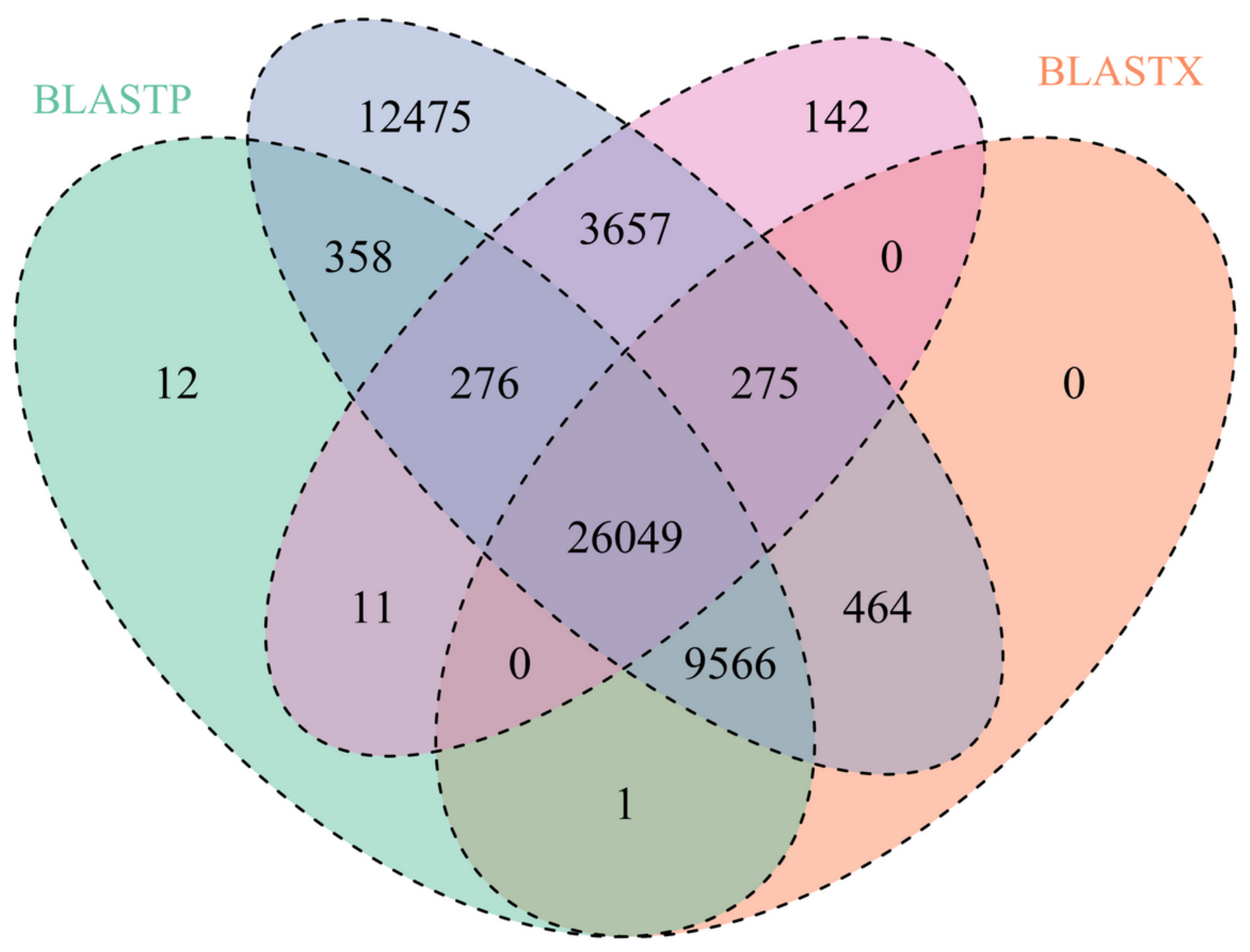

Figure 2

Gene annotation Venn diagram. 

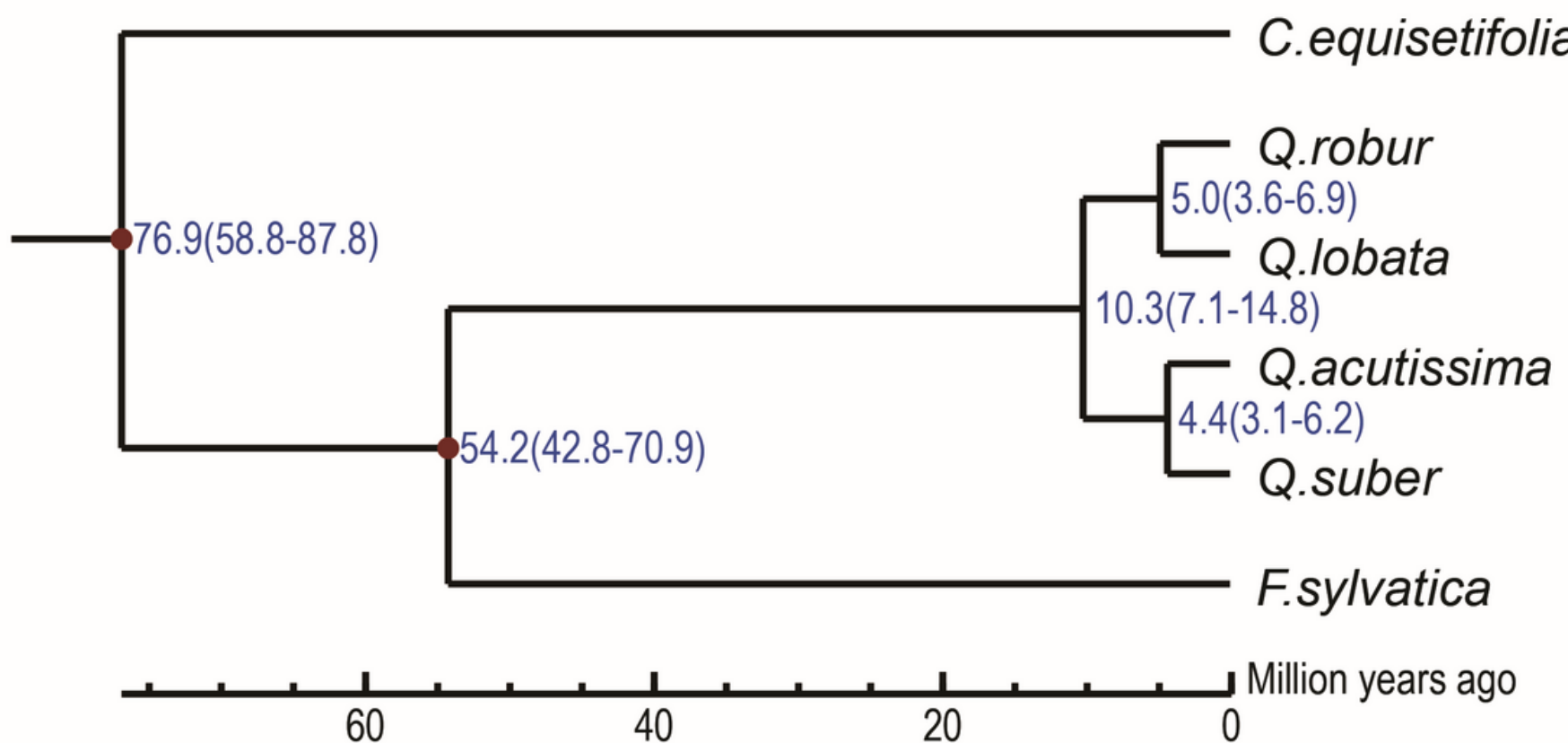

Figure 3

The genome evolution of $Q$. acutissima. 

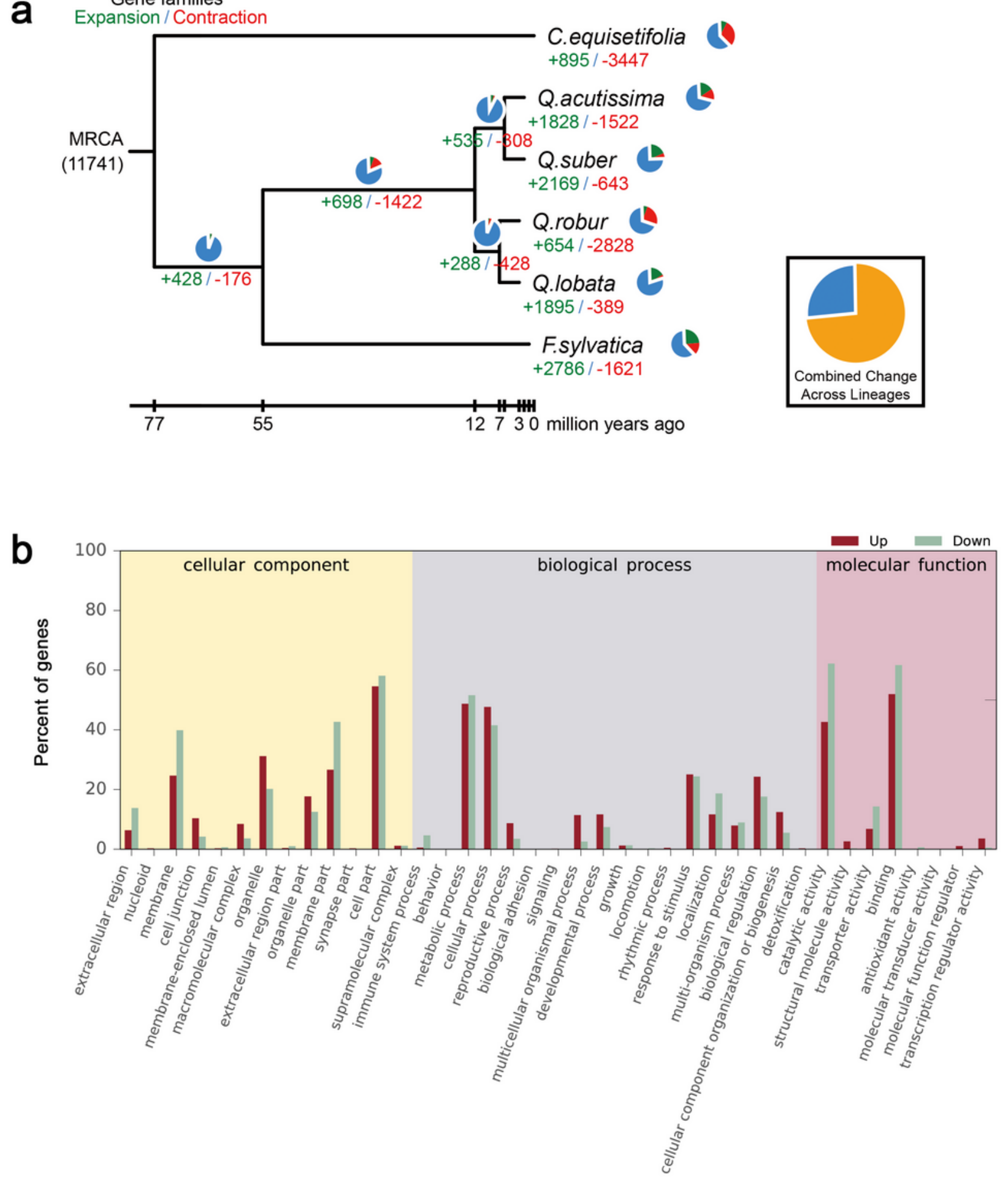

Figure 4

The gene families expansion and contraction analysis. a Family expansion and contraction distribution. The green numbers and red number indicate the number of gene families that underwent expansion and contraction during evolution, respectively. $\mathbf{b}$ The percent of expansion and extraction gene families in Enriched GO terms. Up and down indicates the expansion gene families and extraction gene families, respectively. 


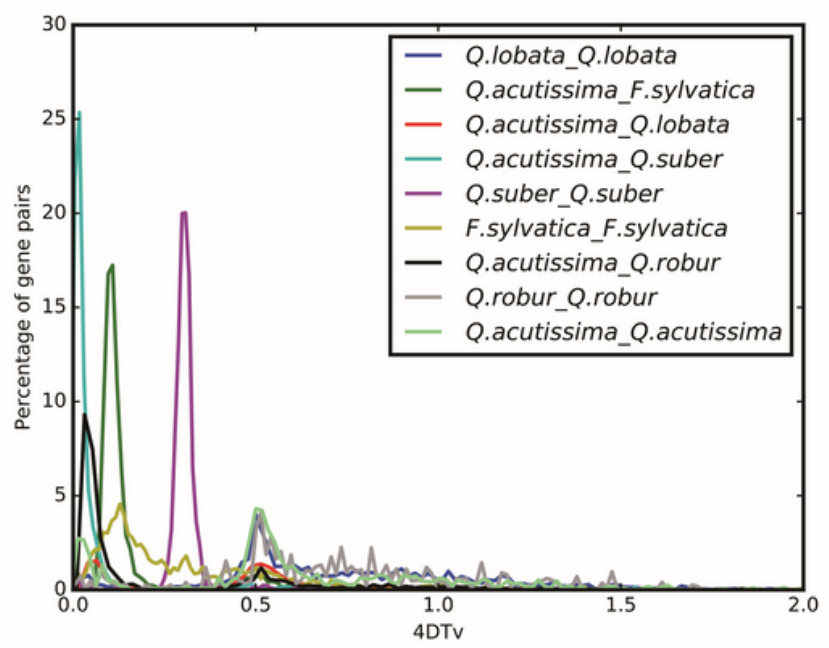

b

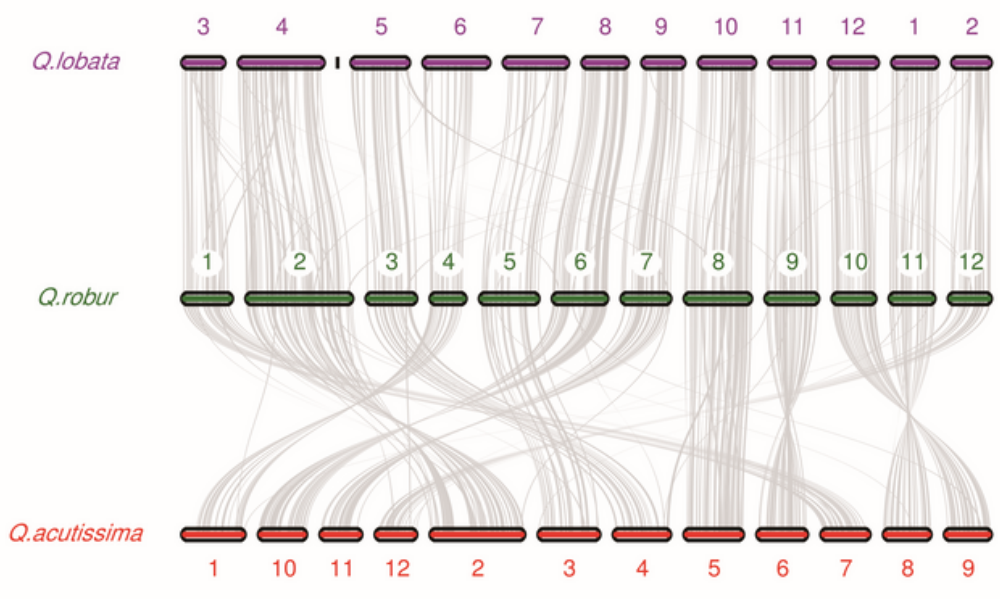

\section{Figure 5}

The WGD events and synteny analysis between $Q$. acutissima and other species. a 4DTv. The abscissa represents the 4DTv value, and the ordinate represents the proportion of genes corresponding to the 4DTV values. b Collinear relationship of $Q$. acutissima, $Q$. lobata and $Q$. robur. The gray line connects matched gene pairs

\section{Supplementary Files}

This is a list of supplementary files associated with this preprint. Click to download.

- FlgureS1ThefrequencycurveofKmerdepth.tif

- FlgureS2TheplottingofGCdepth.tif

- FlgureS3Readcoveragedepthdistributionandintegrityofgenomecoverage.tif

- Flgures4TheVennplotofgenefamily.tif

- TableS1ThestatisticsofBUSCOassessment.docx

- TableS2Thestatisticsofbasecontent.docx

- TableS3Thestatisticsofldentityassessment.docx

- TableS4Thestatisticsofrepeatsequence.docx

- TableS5Statisticalresultsofrepeatedsequenceclassification.docx

- TableS6AnnotationofnoncodingRNAsncRNAs.docx

- TableS7Thenumberofannotatedgenes.docx

- TableS8Quantitativestatisticsofgeneclusteringintofamilies.docx

- TableS9ThestatisticsofExpansionsandContractions.docx

- TableS10Basicstatisticalresultsofgenestructureprediction.docx 